\title{
Ergebnisse geomorphologischer und palynologischer Untersuchungen zum Spätglazial und Frühholozän in den Cuillin Hills auf der Insel Skye (Schottland)
}

\author{
Michael WaltheR *)
}

\author{
Upper Pleistocene, Lower Holocene, geological sections, mapping, glacial features, \\ moraines, kames, oser, drumlins, glacial erosion, pollen diagrams, C 14 dating, paleosols \\ Inner Hebrides, Skye, Glen Sligachan, Harta Corrie, \\ Corrie Lagan, Corrie Ghrunnda
}

\begin{abstract}
Kurzfassung: Es werden Moränen (als rücken-, wall- und kuppenförmige Vollformen ausgebildet), Oser, Kames sowie Formen glazialer Erosion kartiert und in morphologisch abgrenzbare Stadien eingeteilt (A- bis E-Stadien). Blockmoränen, Blockmoränenstreu und Blockmoränenwälle sind charakteristisch für die jüngeren Stadien. An ausgewählten Bohrstellen, die einen unmittelbaren Bezug zu Moränen und anderen glazialen Phänomenen haben, wurden basisnahe Sedimentserien von Bohrkernen palynologisch - kombiniert mit einzelnen radiometrischen Datierungen untersucht. Die Untersuchung ergab deutlich eine spätglaziale (jungtundrenzeitliche) Gletscherbedeckung mit verschiedenen Rückzugsstadien und eine frühholozäne Karvergletscherung. Die bisher wenig beachtete Bodenbildung auf den Moränen konnte Hinweise auf eine Eingruppierung der Moränen in ältere Moränen mit Podsolen, jüngere Moränen mit Rankern und $z$. T. Braunerden und jüngste Moränen mit einer nur sehr schwachen Bodenbildung geben.
\end{abstract}

[Geomorphological Investigations into the Lateglacial and Early Holocene Development in the Cuillin Hills (Isle of Skye, Scotland)]

Abstract: In the course of my survey glaciofluvial forms (terraces, esker hummocks, embankment-like esker ridges and kames) were, for first time, identified and described by means of extensive excavation of the sediments of hummock-shaped features. The intention was to re-examine the validity of the commonly used term "hummocky moraines" as used by SIssONS (1967), according to genetic criteria and the inner structure of these forms.

*) Anschrift des Verfassers: Dr. M. WALther, Freie Universität Berlin, Institut für Physische Geographie, Grunewaldstr. 35, 1000 Berlin 41. Herrn Dr. A. BRANDE, Berlin, bin ich für die vielen Hinweise und Anregungen bei meinen palynologischen Arbeiten zu Dank verpflichtet.
Moraines occur as ridge-, wall- and hummock-shaped forms, whose inner structure sometimes includes meltwater sediments (hummocky moraines in a narrow sense). Block moraines, scattered block debris and block moraine walls are characteristic features of the more recent stages.

Cores were taken at selected sites related to moraines and other glacial features. Pollen analyses of near-base sediment series, together with radiometric measurements provide indications of glacial melt.

Soil formation on the moraines, previously somewhat neglected by researchers, was used to distinguish older moraines with podsols, newer moraines with rankers and sometimes braunerde, and most recent moraines with only little pedogenesis.

\section{Die Arbeitsgebiete und Feldbefunde}

Die Insel Skye liegt zwischen $57^{\circ} 03^{\prime}$ und $57^{\circ} 44^{\prime}$ nördlicher Breite und $6^{\circ} 46^{\prime}$ und $5^{\circ} 38^{\prime}$ westlicher Länge vor der schottischen Westküste zwischen den äußeren Hebrideninseln Uist, Harris und Lewis und dem schottischen Festland (vgl. Abb. 1). Im Bereich der höchsten Erhebungen - den Cuillin Hills (992 m N. N.) - und seinen Vorländern wurden zwei Arbeitsgebiete ehemaliger Gletscherbedeckung ausgewählt: der Bereich des Lota Corrie, Harta Corrie, Glen Sligachan mit seinen Verzweigungen zum Loch Sligachan, Glen Drynoch und Glen Varragill (vgl. Abb. 2 und 3) und als zweites Gebiet die Kare und ihre Vorländer des Corrie Lagan und Corrie Ghrunnda (Abb. 2 und 10). 


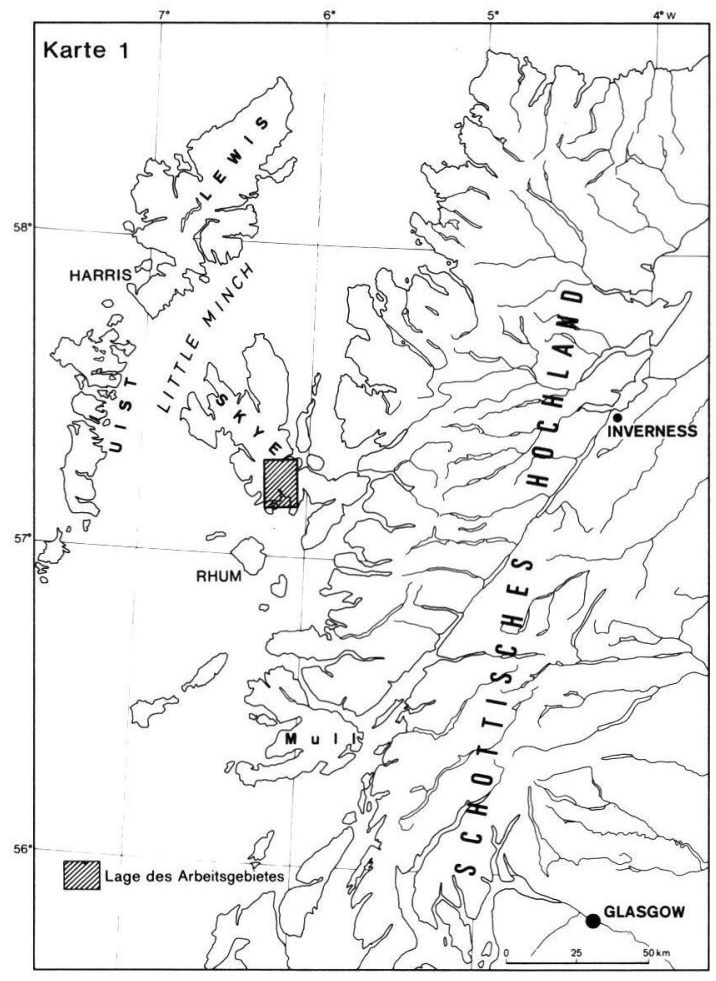

Abb. 1: Lage des Arbeitsgebiets.

Fig. 1: Location of the working area.

\subsection{Der „Sligachan-Gletscher”}

Die granit- und gabbroreichen Moränen im Bereich des "Sligachan-Gletschers" lassen klar darauf schließen, daß der Hauptgletscherstrom aus den Cuillin Hills gekommen ist bzw. durch das Glen Sligachan mit einer mehrfachen Gabelung in das Loch Sligachan, Glen Varragill und Glen Drynoch abfloß. Dabei überflossen die Eismassen die niedrige Paßregion zwischen Glen/Loch Sligachan und Glen Varragill/Drynoch und gestalteten ihre jenseitigen Abflußbahnen zu trogtalähnlichen Talungen.

Beim Eisrückzug bzw. Niedertauen des stationären Eises kam es zu einem phasenweisen Eiszerfall, der zeitlich ins Spätglazial fällt. Es konnten weit vor dem Eisrand der A-Moränenlage des Glen Drynoch Moränenreste gefunden werden, für die keine genauen Datierungsergebnisse vorliegen.

Die A-Moränen im Glen Varragill werden in ihrer äußersten Randlage durch einen mächtigen, das Tal verbauenden Querriegel charakterisiert, hinter dem zur Zeit des Abschmelzens des Eises ein Stausee gelegen haben muß (vgl. Abb. 3 und 4). An der Basis der Bohrung B8 (vgl. Abb. 4) wurden wie bei den
Bohrungen B68 und B69 (WALTHER 1984) glaziolimnische Sand- und Siltserien erschlossen.

Der pollenanalytische Befund vom Bohrprofil B 8 (vgl. Abb. 5) im oberen Glen Varragill zeigt deutlich eine baumfreie Zone im Bereich zwischen $258 \mathrm{~cm}$ und 308 $\mathrm{cm}$, in der Gramineae, Cyperaceae und Myriophyllum in einem tieferen Abschnitt vertreten sind. Der obere Abschnitt dieser Zone ist charakterisiert durch ein Juniperus- und Lycopodium selago-Maximum. Die liegenden Sedimente sind durch geringe Betula-, Salix- und Ericaceae-Werte ausgewiesen. Ab $340 \mathrm{~cm}$ setzen zur Basis hin deutlich geschichtete Silt/Tonund Sandlagen ein. Über der Lycopodium-Zone finden sich Rumex, Gramineae und Cyperaceae reich vertreten, während eine geringe Verbreitung von Corylus einsetzt, die dann in der hangenden Zone zusammen mit Betula immer mehr an Bedeutung gewinnt.

Vergleicht man den palynologischen Befund mit dem Diagramm der auf der SW-Seite der Cuillin Hills gelegenen Lokalität Lochan Coir' A' Ghobhainn von BIRKS (1973), so zeigen sich Parallelen in der Lycopodium-Cyperaceae-Zone und dem Auftreten einer Juniperus-Subzone und der Gramineae-Rumex-Zone im Übergangsbereich zur Betula-Corylus-Zone.

Vergleicht man diese A-Moränen mit den jüngeren Moränen des B- und C-Stadiums, so fallen Übereinstimmungen in der Steilheit der Hänge, dem inneren Aufbau und der Intensität der Bodenbildung auf. Diese Indizien sprechen für eine gewisse Gleichzeitigkeit bei der Entstehung der Formen, wenn man besonders die graduell unterschiedliche Ausprägung der Böden aller Moränen betrachtet. Die A- bis D-Moränen tragen einen Boden mit wenig differenzierten Ah-Bv-C-Profil, während auf Moränendecken außerhalb meines Arbeitsgebietes (z. B. im unteren Glen Varragill, ca. $2 \mathrm{~km}$ südl. Portree) Podsole mit deutlicher Horizontdifferenzierung beobachtet werden konnten.

Die Bohrungen B68 und B69 (WALTHER 1984) liegen bereits hinter dem Außenrand der nächst jüngeren BMoränenlage in der Paßregion zwischen Glen Sligachan und Glen Varragill.

Im Profil B 68 konnte an der Basis mit einem Alter von $10000 \pm 105$ Jahre B.P. (Hv 11936) eine relativ baumarme Zone erbohrt werden, in der krautige Arten wie Myriophyllum (Hinweis auf eine lokale Seephase), Artemisia und Oxyria auftreten (vgl. Abb. 6).

Auch die dicht daneben niedergebrachte Bohrung B69 (vgl. Abb. 7) erreichte an der Basis mit 9930 Jahren B.P. (Hv 11938) ähnlich alte Schichten, nur daß hier bereits erstmalig Corylus und Betula auftreten. 
Karte 4: Topographische Übersichtskarte der Insel Skye

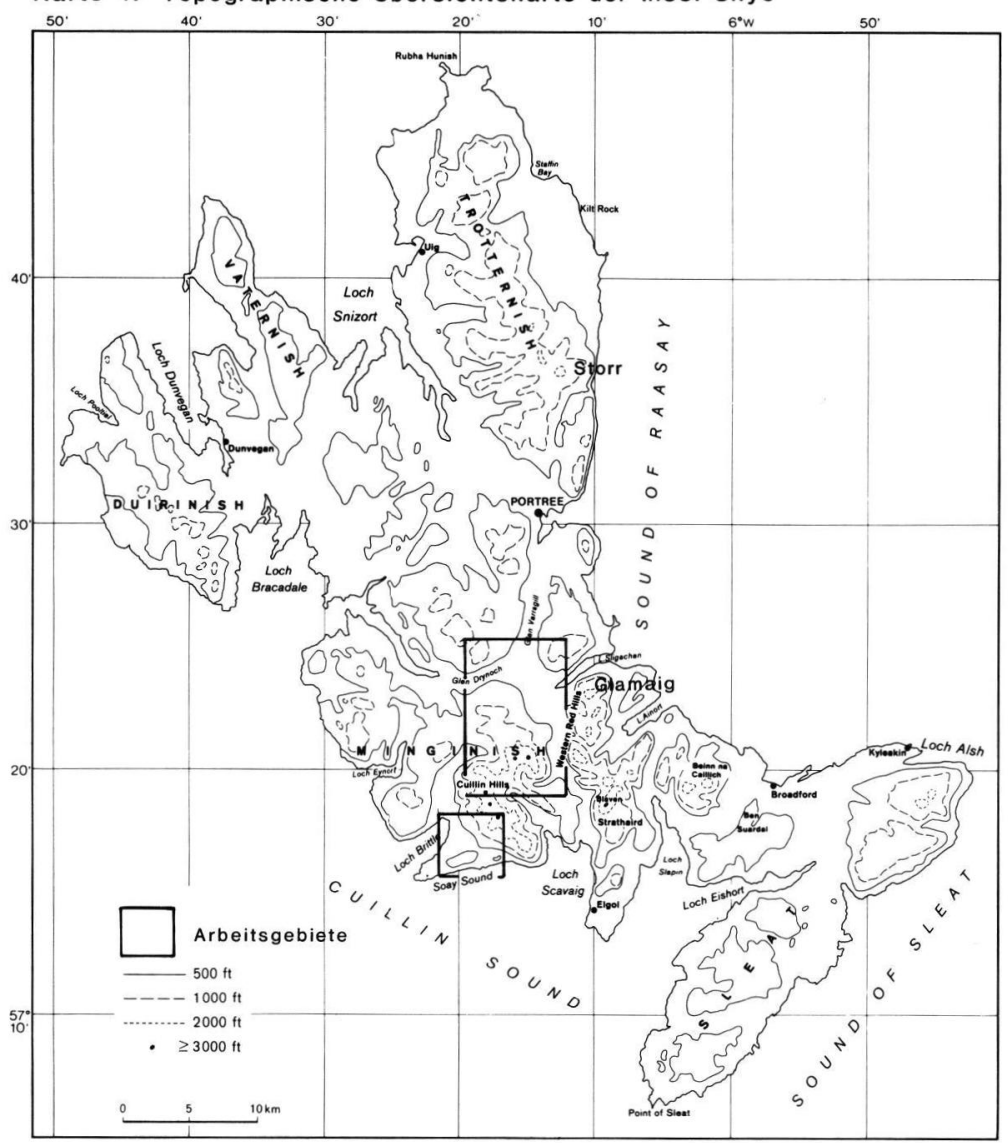

Abb. 2: Topographische Übersichtskarte der Insel Skye.

Fig. 2: Topographical map of the Isle of Skye.
Lycopodium trat in einem Bereich mit einem radiometrisch ermittelten Alter von $8175 \pm 86$ Jahren B.P. (Hv 11937) in größerer Menge auf.

Damit erwiesen sich die Basissedimente als jungtundrenzeitlich. Ich rechne das B1-Stadium zu einer jungtundrenzeitlichen Eisvorstoßphase (vgl. Abb. 3 und 13).

In diesem Zusammenhang ist auf eine zweigliedrige Terrassenabfolge (T1- und T2-Terrasse) im Bereich zwischen den Außenrandlagen des A-Stadiums und B1-Stadiums im Glen Drynoch und Glen Varragill hinzuweisen (WALTHER 1984). Die Lage dieser zwei unterschiedlich hohen Schotterterrassen nahe der Paßregion zwischen Glen Sligachan und Glen Drynoch/ Varragill und die Schotterführung lassen es berechtigt erscheinen, von glaziofluvialen „out-wash”-Terrassen zu sprechen, zumal eine Sortierung von groben, gletscherrandnahen Schottern zu feineren, gletscherrandferneren Kiesen im Verlauf des Glen Varragill feststellbar ist (WALTHER 1984). Die Schmelzwässer haben offenbar während des B-Stadiums die T1- und T2-Terrasse aufgeschüttet und wenig später im ehe- maligen See hinter dem A-Stadium auf der Paßregion erst grobe und dann feinere Sedimente abgelagert.

Die palynologischen Befunde der Bohrungen B68 und B69 und die radiometrischen Datierungen der basisnahen Sedimentserien weisen darauf hin, daß um 10000 Jahren B.P. kein Gletschereis in der Depression oberhalb des B1-Stadiums gelegen haben kann. Die Schichtung von Sand- und Siltlagen (Rhythmit) unterhalb der absolut datierten Mudden läßt vermuten, daß kurze Zeit vor 10000 Jahren B.P. - noch in der Jüngeren Tundrenzeit - die glaziolimnischen Sedimente durch einen periodischen Abtauprozeß von Eismassen entstanden sein müssen. Terrassen sind oberhalb des B-Stadiums nicht mehr vorhanden und ebenso auch keine glaziofluvialen Formen wie Kames oder Oser. Ich nehme an, daß zu Beginn der Jüngeren Tundrenzeit Gletschereis vom „Sligachan-Gletscher” gerade noch die Paßregion erreicht hat.

Glazialmorphologische Zeugnisse des in das Loch Sligachan ausmündenden Glen Sligachan (Oskuppen und Moränenkuppen sowie die Schmelzwasserschlucht des Allt Daraich) deuten auf einen ehemaligen Eis- 


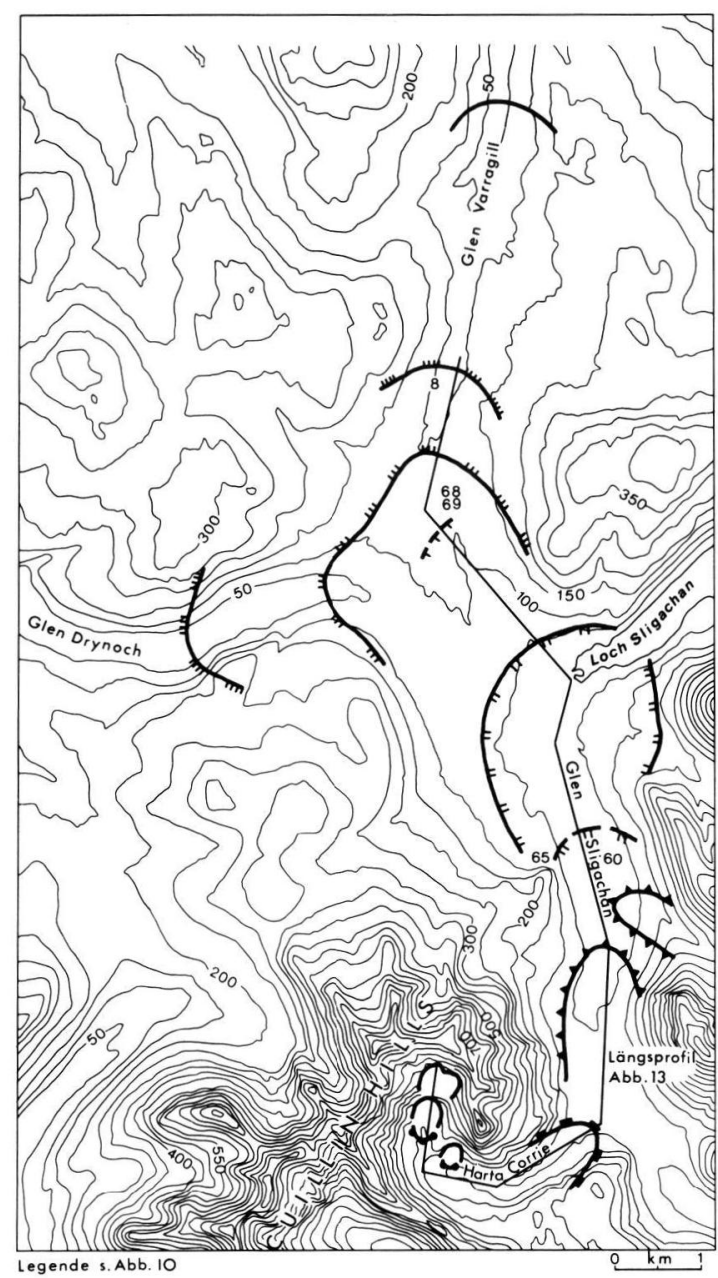

Abb. 3: Gletscherrandlagen vom „Sligachan-Gletscher-System”.

Fig. 3: Ice marginal lines of the "Sligachan-glacier-system".

rand in dieser Region hin (vgl. Abb. 3 und 13). Die gletschertornahen Oskuppen und die von einer niedertauenden und in situ zerfallenden Eisfront herrührenden Moränenkuppen und -wälle auch am Ost- und Westhang des Glen Sligachan sind als Zeugen für dieses B2-Stadium zu nennen (WALTHER 1984: 75 f.f.). In diesem Bereich war es leider nicht möglich, irgendwelche Hinweise auf die Altersstellung durch palynologische oder radiometrische Untersuchungen zu bekommen. Vermutlich gehören die Moränen zu einer Rückzugsphase der Jüngeren Tundrenzeit. Die Böden auf den Moränen zeigen einen gleichen Ausprägungsgrad wie die auf den Moränen der zuvor beschriebenen A- und B1-Randlagen.

In einer großen Depression im Glen Sligachan hinter einem Saum mehrfach gegliederter Moränenkuppen

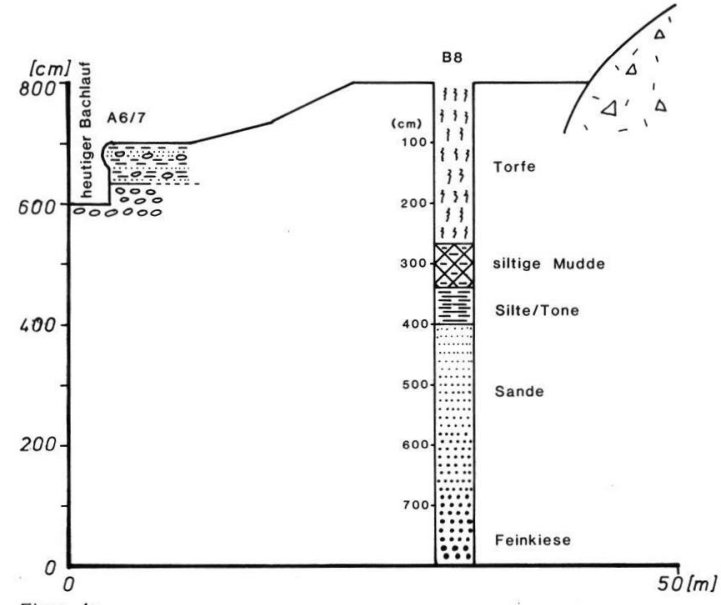

Figur 4:

Bohrprofil B8 und AufschluB A6/7, Glen Varragill

Abb. 4: Bohrprofil B8 und Aufschluß A 6/7, Glen Varragill.

Fig. 4: Core profile B8 and site A6/7, Glen Varragill.

und -wälle (B3-Stadium) konnten zwölf Bohrungen niedergebracht werden (WALTHER 1984: 80 f.f.). Die erreichten Tiefen der Bohrungen und die morphologische Lage dürften für ein Zungenbecken sprechen, das zusätzlich Eismassen noch aus dem Einzugsbereich des „Allt na Measarroch-Gletschers" von SW herbekam (vgl. Abb. 3). Nach den Befunden in mehreren Bohrungen muß sich in diesem Gebiet das Gletschereis am Ende der Jüngeren Tundrenzeit zurückgezogen haben. Das Alter der Basisschichten der Bohrung B 60 mit $9460 \pm 145$ Jahren B.P. und der palynologische Nachweis der Betula-Corylus-Zone zu diesem Zeitpunkt zeigen, daß das Eis schon früher aus diesem Gebiet verschwunden sein muß (WALTHER 1984). Somit dürften die Moränen an den Hängen und der nördlichen Stirnseite der Depression einer dritten jungtundrenzeitlichen Rückzugsphase angehören (vgl. Abb. 3).

Am linken Sligachanhang, westlich der großen Depression, liegt bei einer absoluten Höhe von $200 \mathrm{ft}$. (ca. $61 \mathrm{~m} \mathrm{~N}$. N.) das Dubh Loch, ein kleines Kar mit einem verlandeten See. Eine Muddeprobe von der Basis dieses Seebeckens ergab ein radiometrisches Alter von $10155 \pm 305$ Jahren B.P. (Hv 11935) (vgl. Abb. 8 und 9). Im Hangenden dieser datierten Schicht tritt eine ca. $30 \mathrm{~cm}$ mächtige, ungeschichtete, mit kantigen Bruchstücken durchsetzte Schuttdecke oder Moräne auf, im Liegenden ein jungtundrenzeitlicher Abschnitt i. S. einer Lycopodium-Cyperaceae-Zone (vgl. Abb. 9), während zwischen der datierten Schicht und der Schuttdecke oder Moräne sich eine BetulaCorylus-Zone nachweisen ließ. Dieser Befund weist eindeutig darauf hin, daß erstens in der Jüngeren Tundrenzeit hier Gletschereis gelegen haben muß, 


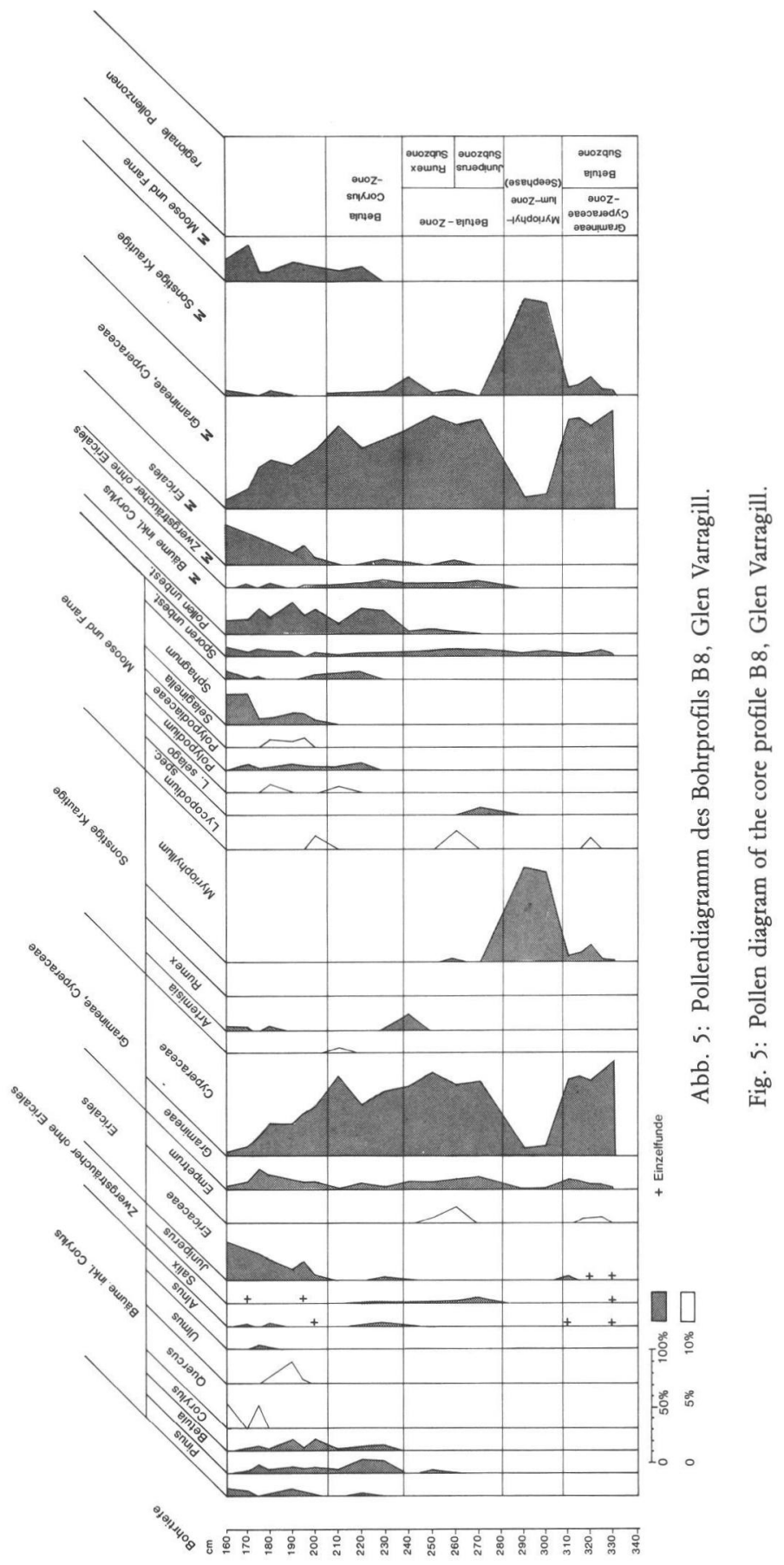




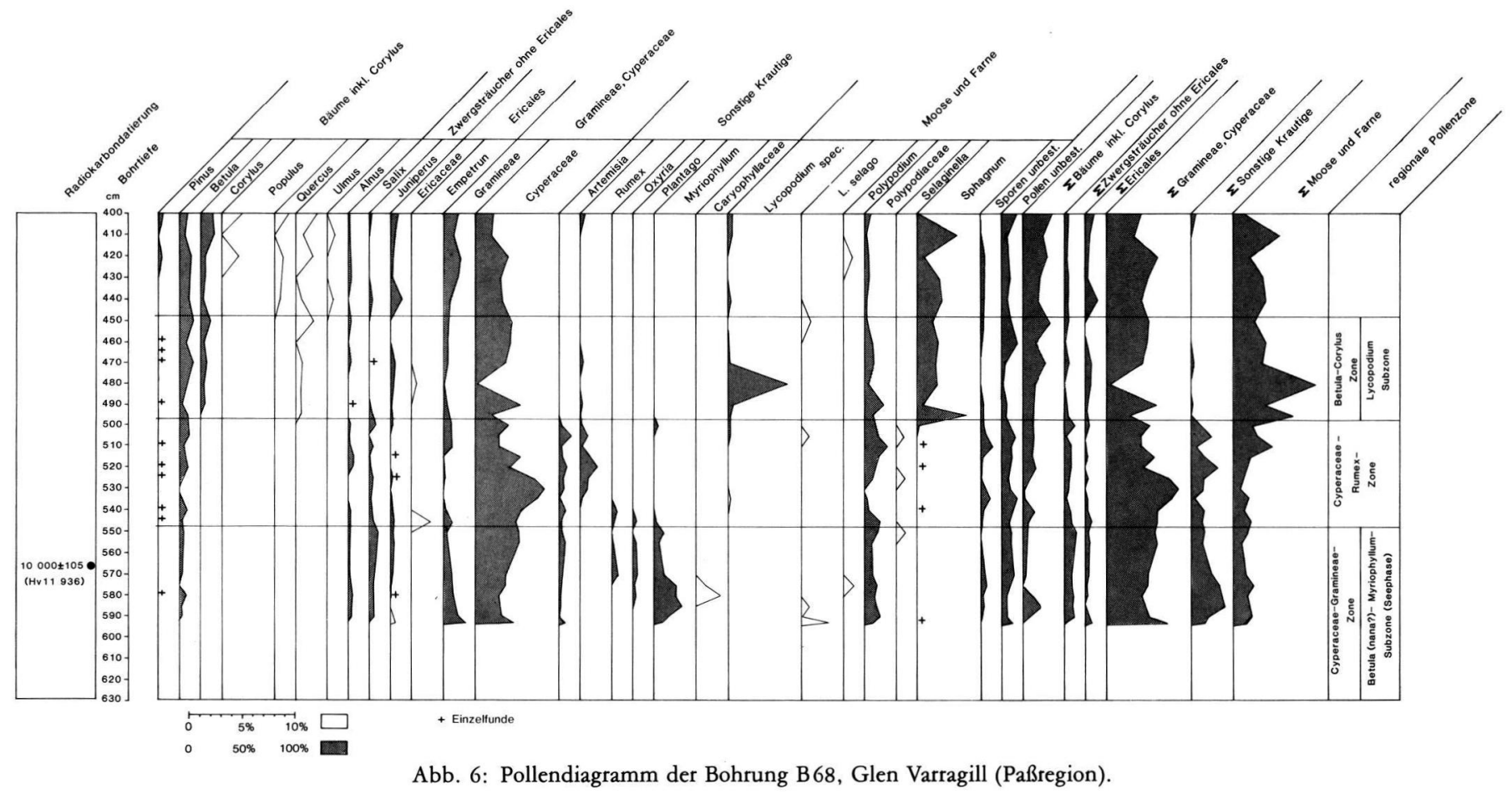

Fig. 6: Pollen diagram of the core profile B68, Glen Varragill. 


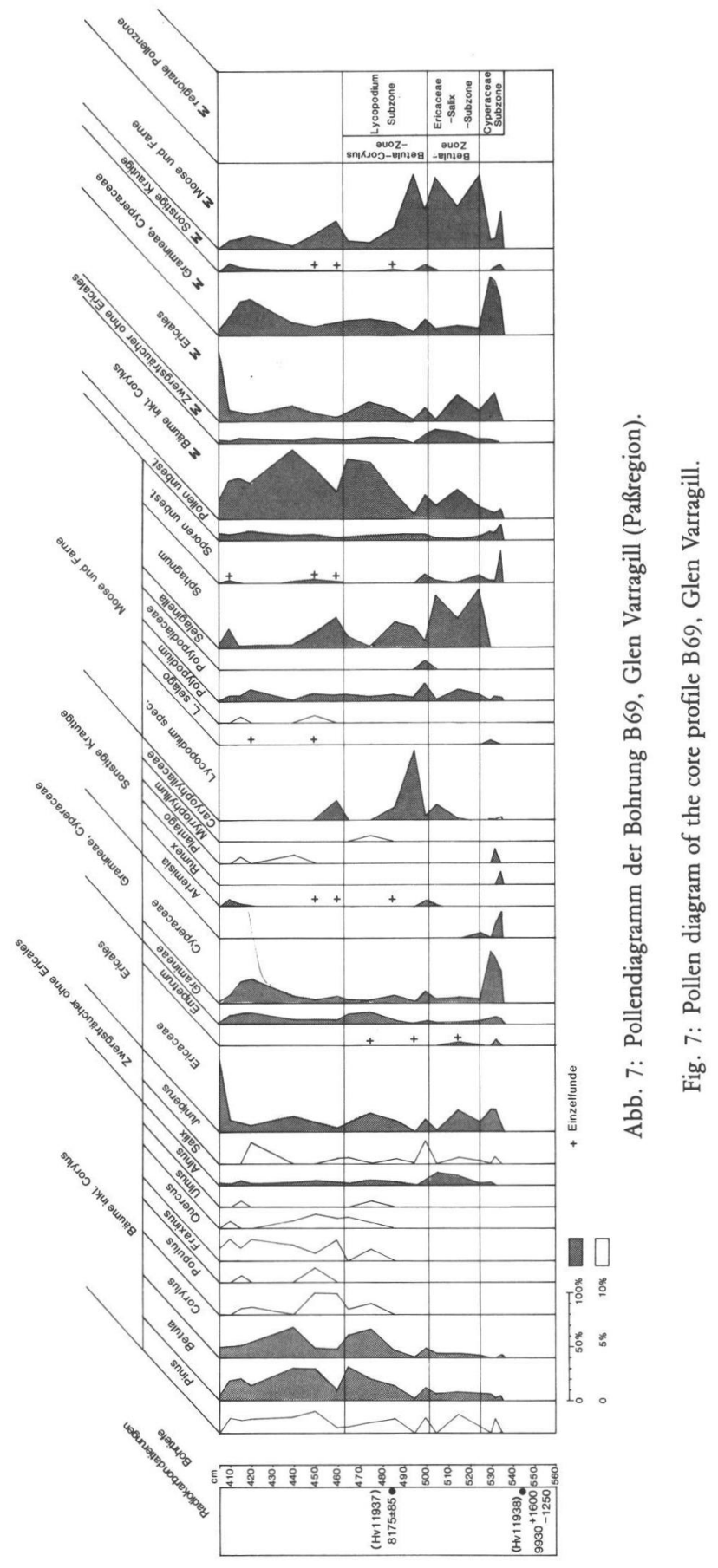


Tab. 1: Übersicht der Radiokohlenstoffdatierungen ${ }^{1}$ )

Alter B. P.

Pr.-Nr.

Profil

datierte Substanz

$\begin{array}{llll}3883 \pm 70 & \text { Hv } 11933 & \text { B } 64 \text { (Glen Sligachan) } & \text { Mudde } \\ 4310 \pm 110 & \text { Hv } 11930 & \text { B } 45 \text { (Glen Sligachan) } & \text { Holzkohle } \\ 4705 \pm 115 & \text { Hv } 11934 & \text { B } 64 \text { (Glen Sligachan) } & \text { Holzkohle } \\ 7780 \pm 180 & \text { Hv } 11931 & \text { B } 57 \text { (Corrie Lagan) } & \text { Mudde } \\ 8175 \pm 85 & \text { Hv } 11937 & \text { B } 69 \text { (Glen Varragill) } & \text { Holzkohle } \\ 9460 \pm 145 & \text { Hv } 11932 & \text { B } 60 \text { (Glen Sligachan) } & \text { Mudde } \\ 9930 \pm 1600 & \text { Hv } 11938 & \text { B } 69 \text { (Glen Varragill) } & \text { Mudde } \\ 10000 \pm 1250 & \text { Hv } 11936 & \text { B } 68 \text { (Glen Varragill) } & \text { Mudde } \\ 10155 \pm 305 & \text { Hv } 11935 & \text { B 65 (Loch Dubh) } & \text { Mudde }\end{array}$

welches während der Rückzugsphasen ausschmolz und daß zweitens hier offenbar schon im Holozän sich nochmals deutlich ein kühleres bis kaltes Klima abzeichnet, eventuell präborealen oder borealen Alters.

Verfolgt man nun das Glen Sligachan aufwärts, so ergeben sich aus der Geländeaufnahme (WALTHER 1984) ab dem folgenden C-Stadium Unterschiede in Hinsicht auf das glazialmorphologische Formeninventar. Moränen, hier an strukturelle Vorzeichnungen des Untergrundes angelehnt, treten als morphologische Positivformen in den Hintergrund und sind nur im nördlichen und südlichen Teil dieses Stadiums vertreten (vgl. Abb. 3). Wesentlich häufiger und landschaftsprägend treten Kames in verschiedenen morphogenetischen Erscheinungsformen auf (WALTHER 1984: Fig. 34). Sie sind als Leitformen der niedertauenden Eisfront während der vierten jungtundrenzeitlichen Rückzugsphase zu deuten. Die gleichartige Ausprägung der Böden im Vergleich zu den Böden auf Moränen des B-Stadiums spricht hier für ein jungtundrenzeitliches Alter.

Zum C-Stadium rechne ich auch den ehemaligen Gletscherlobus, der in dem von SW spitzwinklig auf das Glen Sligachan ausmündenden Talzug des Allt na Measarroch gelegen haben muß (vgl. Abb. 3). Die Moränen und dammförmigen Oser in seinem Mündungsbereich sind aufgrund der Bodenbildung und der unmittelbar vor dem heutigen Schwemmkegel des

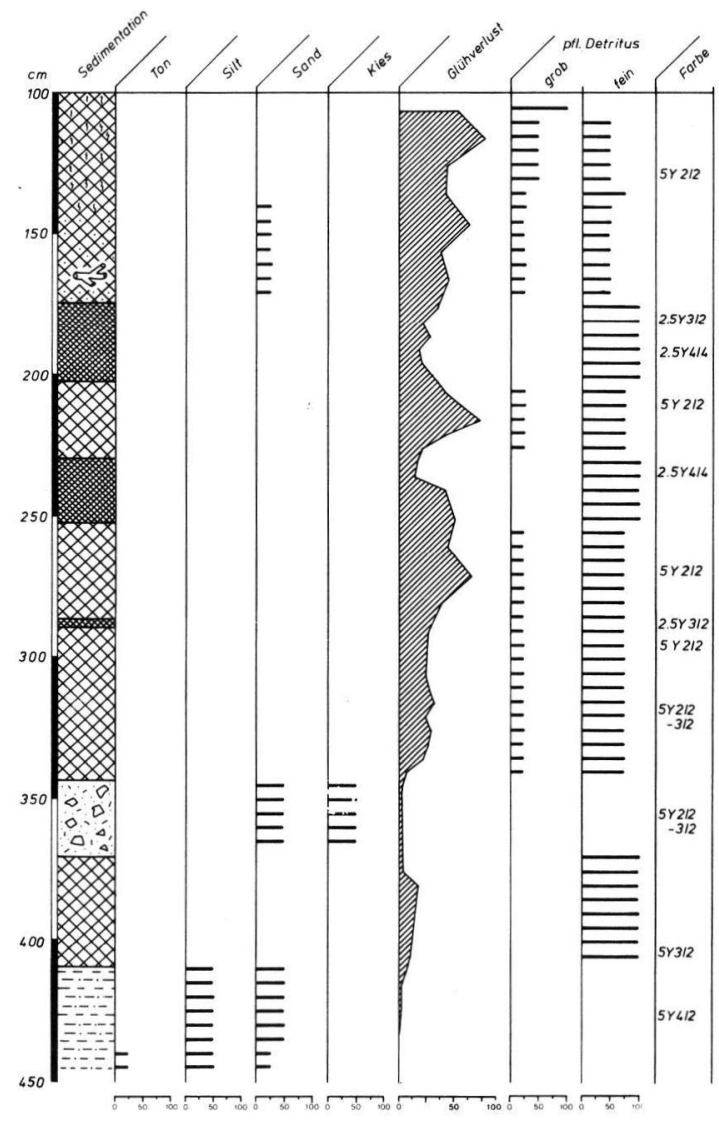

1) Für die Datierung von neun Proben bin ich Herrn Prof. Dr. M. A. GEYH, Hannover, zu Dank verpflichtet.

Abb. 8: Bohrprofil B 65, Dubh Loch.

Fig. 8: Core profile B65, Dubh Loch. 
Baches niedergebrachten Bohrungen als spätjungtundrenzeitlich einzustufen.

Die letzte und fünfte jungtundrenzeitliche (oder jüngere) Rückzugsphase (D-Stadium) ist morphologisch charakterisiert durch eine Anhäufung von Blöcken und wenigen Kames im Verlauf der Taltiefenlinie des Harta Corrie (vgl. Abb. 3). Die Außenbegrenzung dieses D-Stadiums ergibt sich $\mathrm{u}$. a. auch aus den $\mathrm{Pa}$ rallelisierungsversuchen mit den Karen auf der Südwestseite der Cuillin Hills, worauf im Anschluß näher eingegangen wird. Im Harta Corrie ergaben sich leider keine Anhaltspunkte für das Alter des D-Stadiums.

Die jüngsten, ausgewaschenen Blockmoränenwälle wurden in der nordwestlichen Fortsetzung des oberen Harta Corrie gefunden (vgl. Abb. 3). Auch hierzu gibt es ein Parallelbeispiel in den Karvorfeldern der Südwestseite der Cuillin Hills. Aufgrund der Formenfrische muß angenommen werden, daß diese Moränen nicht einer periglazialen Klimaphase ausgesetzt waren, die sich wie bei den anderen älteren Moränen oder glaziofluvialen Formen hätte hangverflachend ausgewirkt haben müssen. Die Böden sind auf diesen

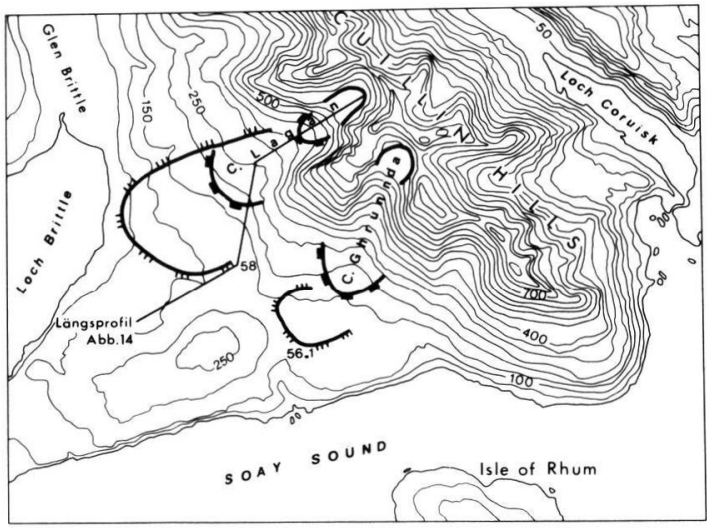

Zeichenerklärung zu den Abb. 3 und 10

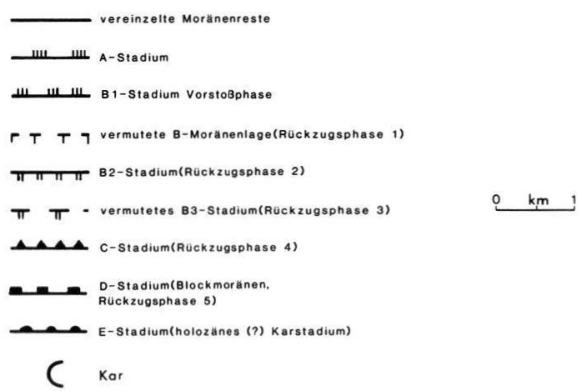

Abb. 10: Gletscherrandlagen im Corrie Lagan und Corrie Ghrunnda.

Fig. 10: Ice marginal lines of Corrie Lagan and Corrie Ghrunnda.
Moränenwällen im Gegensatz zu den vorher genannten Moränen nicht oder nur sehr schwach (allenfalls mit einem A-C-Profil vom Typ eines Ranker) entwickelt. Eine Bohrung ließ sich nicht abteufen, da die Moränenblockstreu auch in den Depressionen zu mächtig war.

\subsection{Die ehemaligen Gletscher im Corrie Lagan und Corrie Ghrunnda}

In den Vorfeldern der beiden untersuchten Kare auf der Südwestseite der Cuillin Hills und in den Karen selbst zeigt sich folgende Situation:

Grundsätzlich war aufgrund der Reliefsituation davon auszugehen, daß die ehemaligen Gletscher, in einem steilen präglazialen Relief eingetieft, nur einen relativ kurzen Weg im Sinne eines Talgletschers wie im Glen Sligachan zur Verfügung hatten und dann in späteren Abtauphasen ihre glazialen Hinterlassenschaften nicht weit entfernt voneinander ablagern konnten. Man mußte also von vornherein mit einer viel dichteren Staffelung von Moränen und anderen Phänomenen rechnen. Die Horizontaldistanz zwischen dem Corrie Lagan und der heutigen Küstenlinie beträgt nur 4,2 km, während zwischen der nordwestlichen Fortsetzung des oberen Harta Corrie und dem oberen Ende des Glen Varragill 13,7 km liegen.

Die Bohrstelle von BIRKS (1973) im Lochan Coir' A' Ghobhainn liegt außerhalb der von mir als A/B-Stadium bezeichneten Moränenrandlage; eine radiometrische Datierung basisnaher Sedimente ergab ein Alter von $10254 \pm 220$ Jahren B.P. (Q-955). Aufgrund der Sedimentführung und des Pollendiagramms von diesem Profil kann davon ausgegangen werden, daß gegen Ende der Jüngeren Tundrenzeit kein Gletscher mehr dort gelegen haben kann; jedoch kann nicht ausgeschlossen werden, daß zu diesem Zeitpunkt noch innerhalb der A/B-Randlage des Corrie Lagan Eis vorhanden war (vgl. Abb. 10).

Eine Datierung und die palynologische Bearbeitung der Bohrprofile B57 und B58 weisen für die Basisserien dieser knapp außerhalb der Randlage gelegenen Depression ein Alter von $7780 \pm 180$ Jahren B.P. (bei $B$ 57) und fragmentarisch eine jungtundrenzeitliche Pollenzone (bei B 58) aus (vgl. Abb. 11). Dieser Befund deutet darauf hin, daß eine vorausgehende, wahrscheinlich jungtundrenzeitliche Vergletscherung des Vorlandes im Corrie Lagan bis auf knapp $50 \mathrm{~m}$ N.N. gegangen ist (WALTHER 1984).

Im Vorland des Corrie Ghrunnda dürfte zeitgleich das Loch Meachdannach am Außenrand des dortigen jungtundrenzeitlichen Gletschers gelegen haben. Die Bohrungen B 56 und B 56.1 (vgl. Abb. 12) zeigen $z$. T. deutliche Pollenspektren einer jungtundrenzeit- 


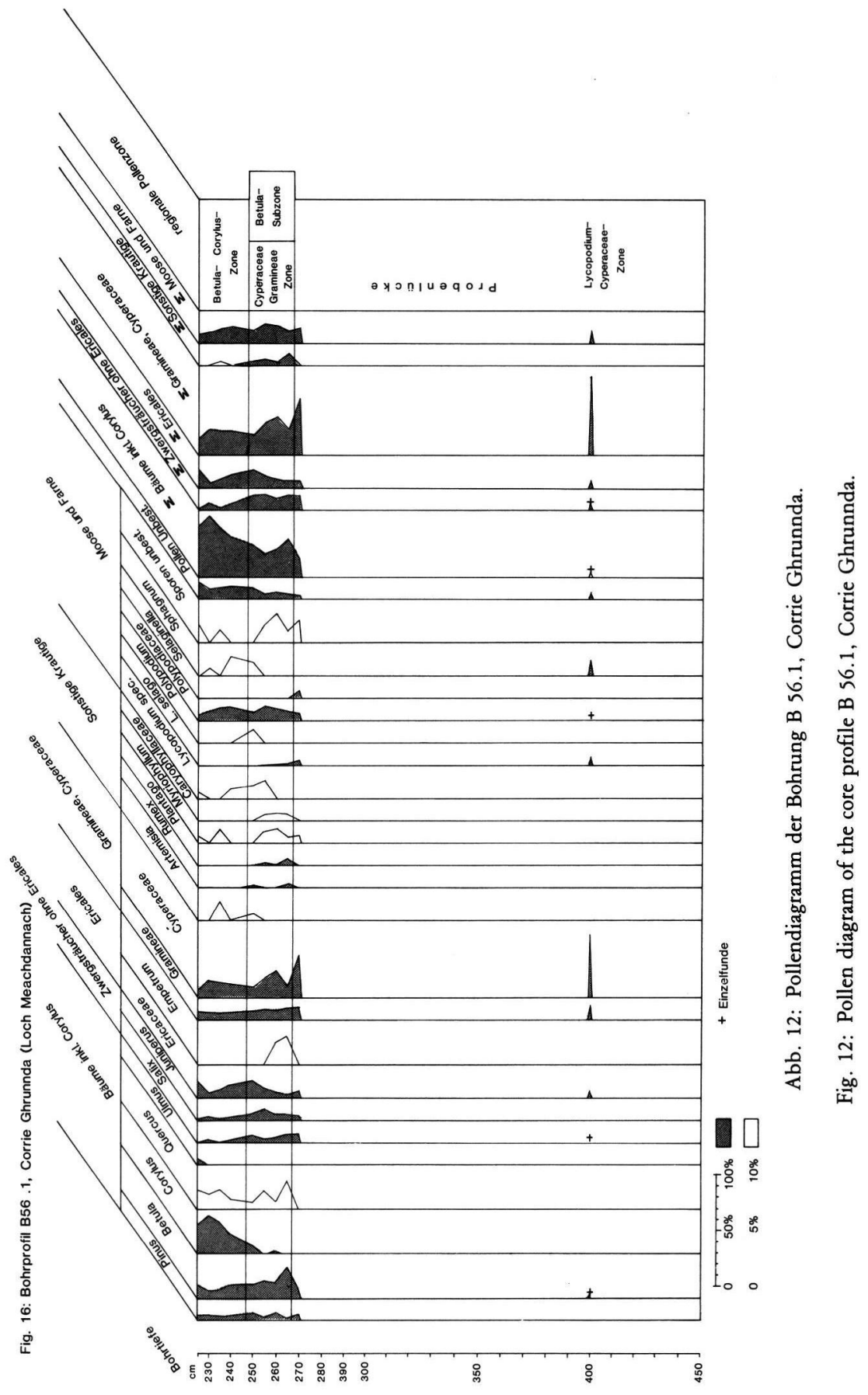




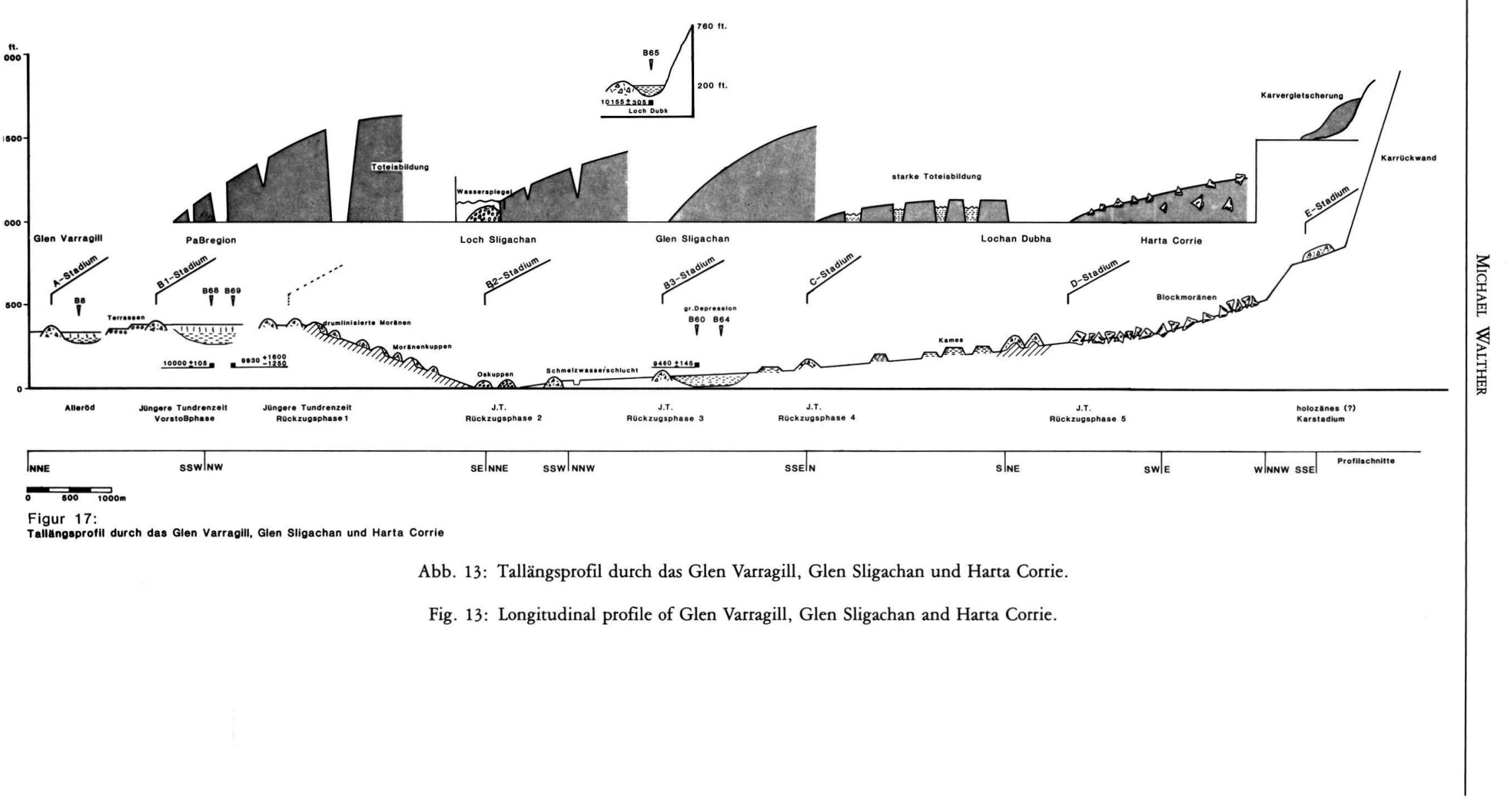




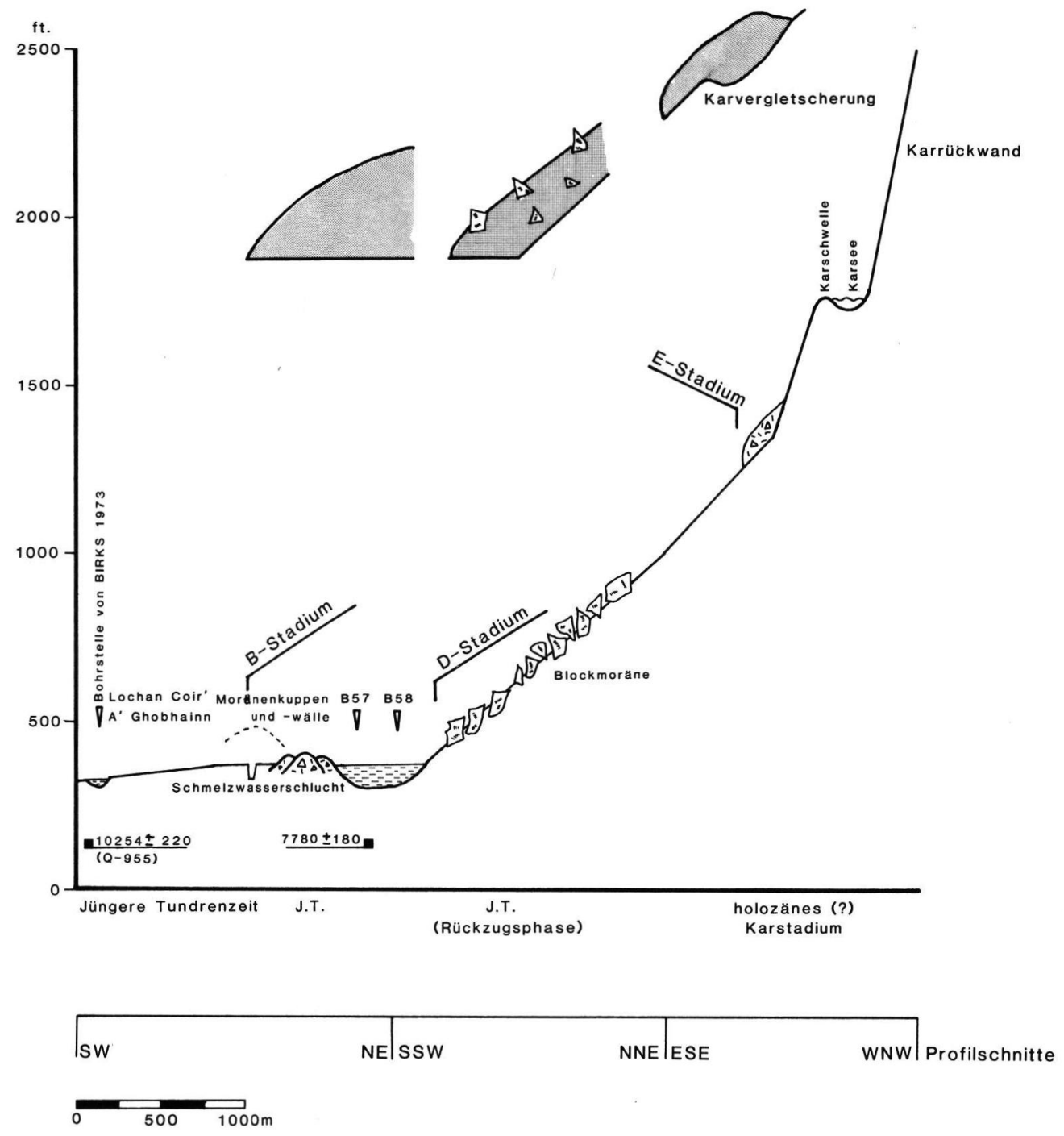

Abb. 14: Längsprofil durch den Corrie Lagan.

Fig. 14: Longitudinal profile of Corrie Lagan.

lichen Pollenzone in den basalen, organogenen Sedimentserien, wobei die minerogenen Basisserien im Liegenden auf direkte Eisnähe schließen lassen.

\section{Diskussion}

Das Alter der morphologisch sich deutlich abhebenden Blockmoränen des D-Stadiums in beiden Teilarbeitsbereichen (Harta Corrie zum Glen Sligachan gehörig, Corrie Lagan und Corrie Ghrunnda) könnte m. E. an die Grenze zwischen Jüngerer Tundrenzeit und Präboreal zu stellen sein, was allerdings der Ver- mutung anderer Autoren (u. a. SISSONS 1977) entgegenstehen würde, die diese Moräne bei ca. $150 \mathrm{~m}$ N.N. als jungtundrenzeitlich oder noch älter (BIRKS 1973) ansehen. Auch zeigen sich gute Übereinstimmungen in der Intensität der Böden zwischen den einzelnen Lokalitäten mit Moränen des D-Stadiums. Geht man neben den morphologischen Befunden nach der Höhenlage dieser Blockmoräne, so ergeben sich für den Harta Corrie mit seiner leeseitigen, östlichen Lage tiefere Werte als die Lage der Blockmoräne auf der West- und Südwestseite der Cuillin Hills; erwartungsgemäß reichten zu gleicher Zeit (vermutlich spätere Jüngere Tundrenzeit oder Präboreal) die 
strat.

Bezeichnung morphologische Erscheinungsformen

räumliche Verteilung

Altersstellung

A

morphologisch deutlich sichtbare wall- und kuppenförmige Moränen, z. T. als Moränenkomplex ausgebildet ( = Anhäufung moränaler Ablagerungen auf relativ engem Raum mit unruhiger Oberfläche, auf der vereinzelt Moränenblöcke liegen; Osrücken; zwei verschieden hohe Terrassenniveaus ( $\mathrm{T} 1$ und T2-Terrasse)

B regelhaftes Auftreten kuppiger Satzmoränen ("hummocky moraines"); morphologisch sind vier verschiedene Halte zu unterscheiden: eine Vorstoßphase und drei Rückzugsphasen; unvermitteltes Auftreten von Blöcken auf den Moränen; hangparallele „Moränenterrassen”; wall- und kuppenförmige Kames (selten); Oser

typisches Auftreten wallförmiger Moränen; mehrere wallförmige Wallmoränen an strukturelle Vorzeichnungen angelehnt; Hauptverbreitung von Kames

Blockmoränen mit z. T. wallartigem Charakter; Riesenblöcke; errechnete Schneegrenze: $592 \mathrm{~m}$ auf der W-Seite; $509 \mathrm{~m}$ auf der E-Seite seltene Moränenschuttwälle; dreiseitig geschlossene Kare, Rundhöckerfluren; seltener Erratika; ganz geringe Bodenbildung; errechnete Schneegrenze: $742 \mathrm{~m}$ auf der W-Seite; $589 \mathrm{~m}$ auf der E-Seite
Glen Drynoch, ca. 2 km SW des Gehöfts Crossal; Glen Varragill am oberen Anfang des Talzuges; auf der Westseite Blockstreu im äußeren Corrie Lagan-Vorland

B 1: Moränen der Paßregion zwischen Glen Varragil und Glen/Loch Sligachan; ca. 500 m E des Gehöfts Crossal;

vermutete B-Randlage: am südöstlichen Rand der Paßregion

B2: Randlage des Loch Sligachan

B3: Randlage am NNE-Rand der großen Depression im Glen Sligachan

im Glen Sligachan vom Allt na Measarroch aufwärts bis zum Lochan Dubha; auf der Westseite vereinzelt Moränenkuppen und -wälle außerhalb der

D-Moränen

unterer und mittlerer Harta Corrie; Corrie Lagan; Corrie Ghrunnda

oberer Teil des Harta Corric (Coire na Clach, Lota Corrie); Corrie Lagan, Corrie Ghrunnda spätglaziale

Altersstellung:

Jüng. Tundrenz.

oder älter;

Terrassen haben

möglicherweise

jungtundrenzeitl.

Alter.

Jüng. Tundrenz.

Jüng. Tundrenz.

Jüng. T./Präboreal

BIRKS (1973):

älter als 12500

SisSONS (1977):

Loch Lomond

Read.

Holozän

(Prä-

boreal ?);

BIRKS (1973):

Loch Lomond

Read. 
Tab. 3: Vergleichende Stratigraphie von Moränenrandlagen in Nord-, Mittel- und Westeuropa im Spätglazial und Frühholozän.

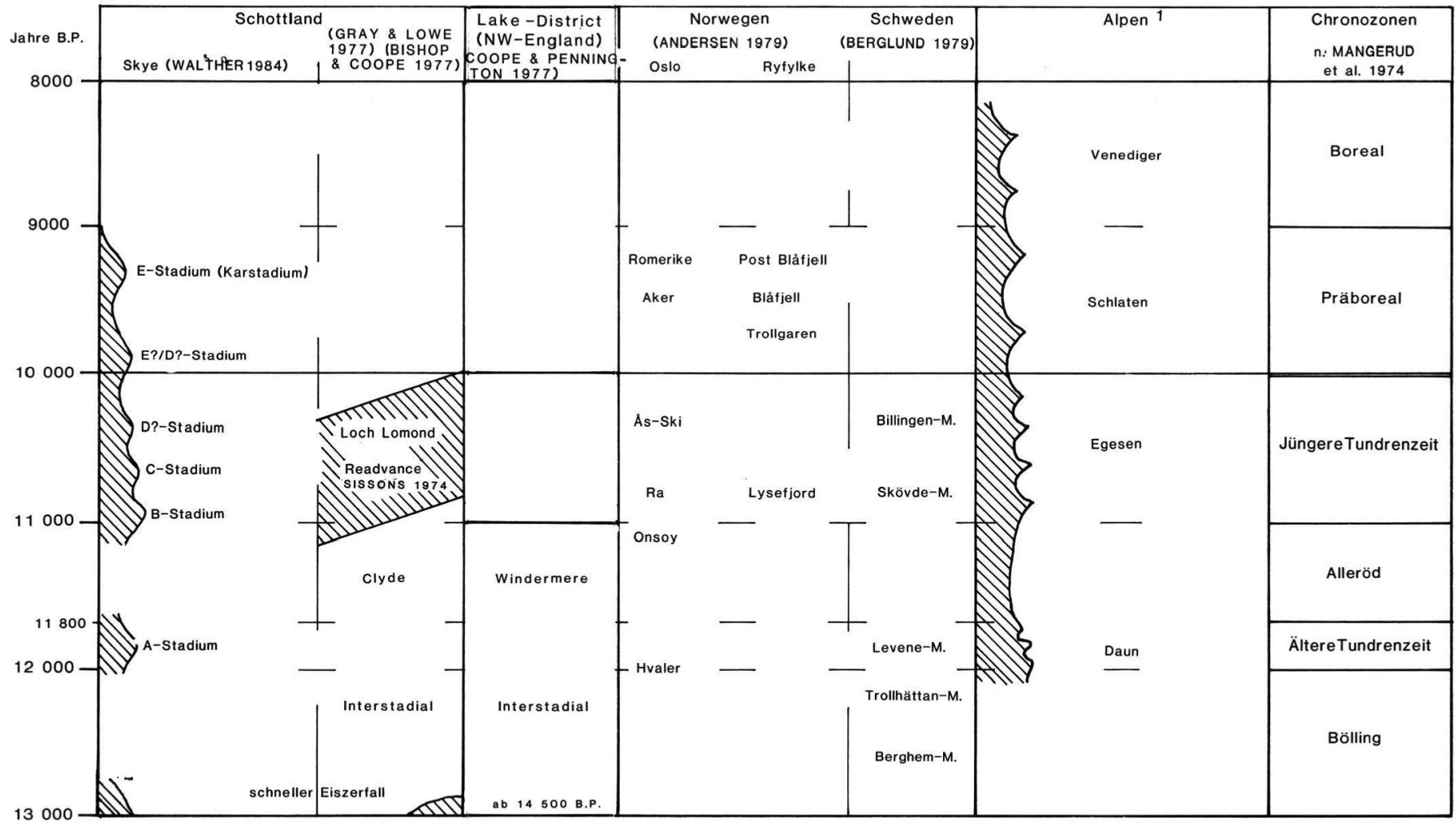

1) In Anlehnung an Heuberger (1966, 1968), Patzelt (1973), Gross (1974), Kerschner (1976), Beeler (1977), Maisch (1981), RenNer (1982) und VAN HUSEN (1983). 


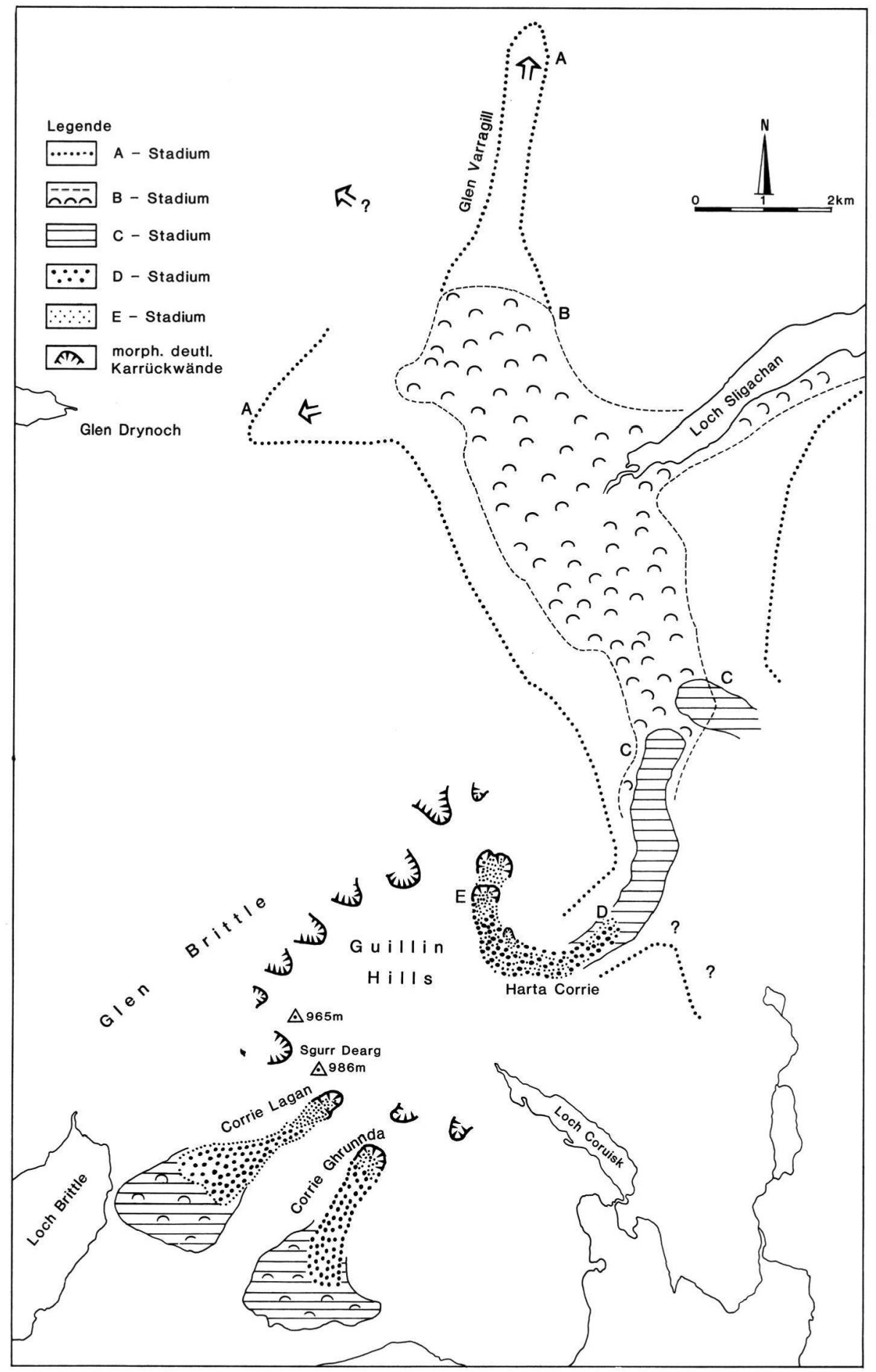

Abb. 15: Rekonstruierte Eisbedeckung im Spätglazial und Frühholozän an drei Beispielen in den Cuillin Hills.

Fig. 15: Reconstruction of lateglacial and early Holocene ice cover illustrated by three examples in the Cuillin Hills. 
Gletscher auf der Ostseite tiefer hinab als auf der West- und Südwestseite des Gebirgsmassivs. Dieser Befund stimmt gut mit den Ergebnissen für die Nordküste Schottlands (SISSONS 1977a) und den LakeDistrict (COOPE \& PENNINGTON 1977) überein.

Sucht man nach den jüngsten im Bereich der nordwestlichen Fortsetzung des oberen Harta Corrie noch deutlich vorhandenen Moränen des E-Stadiums, so findet man im Corrie Lagan bei ca. $430 \mathrm{~m} \mathrm{~N}$. N. unterhalb der Karschwelle eine recht frisch wirkende Moräne, die keine Entsprechung im Corrie Ghrunnda hat. Diese dem E-Stadium zuzurechnende Moräne trägt keinen Boden oder nur stellenweise einen schwach entwickelten Ranker und weist nach Hangwinkelmeßreihen den steilsten Hangwinkel aller Moränen überhaupt auf. Auch hier liegt im Vergleich zum Harta Corrie die Moräne auf der West- und Südwestseite höher als auf der Ostseite. Wie bereits ausgeführt, macht diese Moräne einen so frischen Eindruck, daß sie mit historischen Gletscherständen der Alpen vergleichbar erscheint. BIRKS (1973) vermutet bei dieser Moräne ein Loch Lomond-zeitliches Alter (vgl. Tab. 3).

Vergleicht man nun benachbarte Großräume wie etwa Norwegen, Schweden, das alpine Vereisungsgebiet oder britische Beispiele spätglazialer Vergletscherung, so lassen sich parallele Entwicklungen in den verschiedenen Gebieten in den letzten 14000 Jahren feststellen (vgl. Tab. 3).

Nach meinen Untersuchungsergebnissen muß man für den ehemaligen Sligachan-Gletscher eine Länge von ca. $13 \mathrm{~km}$ in der Jüngeren Tundrenzeit annehmen; nach der bisher allgemein angenommenen totalen Eisfreiheit seit dem Beginn des Holozäns für Schottland würde dies bedeuten, daß diese gewaltige Eismasse in einem relativ kurzen Zeitraum bis 10000 Jahre B.P. verschwunden gewesen sein muß.

Ferner sprechen die theoretischen Berechnungen über den Verlauf der klimatischen Schneegrenze (vgl. MESSERLI 1967; WILHELM 1974) für eine jüngere Karvergletscherung im Präboreal oder sogar noch im Boreal. Es würde heute eine Temperaturerniedrigung von nur 1,7 Grad C genügen, um die von MESSERLI für die gemäßigten Breiten genannten 4,5 Grad C Julimitteltemperatur für die klimatische Schneegrenze in den Gipfellagen der Cuillin Hills zu erreichen, wobei ausdrücklich auf bestimmte Fehlermöglichkeiten in der Schwankung der Schneegrenze hinzuweisen ist. Nimmt man die Erkenntnisse über die Niederschläge sowie die Massenerhebung der Cuillin Hills hinzu, so ergeben sich denkbar günstige Bedingungen für die spätglaziale und frühholozäne Gletscherentwicklung in meinem Arbeitsgebiet.
Nimmt man für die Berechnung der vorzeitlichen Schneegrenze (VON HÖFER 1879) als Bezugsgröße die Randlage des A-Stadiums beim ehemaligen SligachanGletscher, wobei die Randlage im Loch Sligachan unter der heutigen Meeresbedeckung gelegen haben muß, so kommt man auf Werte von $427 \mathrm{~m} \mathrm{~N}$. N. für die Maximalrandlage der Jüngeren Tundrenzeit (auf der Südwestseite entspricht dies $488 \mathrm{~m} \mathrm{~N}$. N.). Diese Werte stimmen annäherungsweise mit den von SISSONS (1980) berechneten Werten für das schottische Hochland und die Inneren Hebriden überein.

Nach den pedologischen, sedimentologischen, palynologischen und radiometrischen Ergebnissen sowie aufgrund der Kartierungsbefunde ist davon auszugehen, daß ein jungtundrenzeitliches Alter für das AStadium, sicher aber ab dem B-Stadium in Betracht gezogen werden muß. Ferner wurde deutlich, daß nach der Jüngeren Tundrenzeit noch in der nordwestlichen Fortsetzung des oberen Harta Corrie und unterhalb der Karschwelle des Corrie Lagan jüngste Moränen vom Gletschereis abgelagert wurden, für die ein holozänes Alter anzunehmen ist.

\section{Schriftenverzeichnis}

Andersen, B. G. (1979): The Deglaciation of Norway, 15000-10000 B.P. - Boreas, 8: 79-89; Oslo.

Anderson, F. W. \& Dunham, K. C. (1966): The Geology of Northern Skye. - Mem. geol. Surv., 120 S; Edinburgh.

BEELER, F. N. (1977): Geomorphologische Untersuchungen am Spät- und Postglazial im Schweizerischen Nationalpark und im Berninapaßgebiet. - in: Ergebnisse der wissenschaftlichen Untersuchungen im Schweizerischen Nationalpark, 15: 131-276; Liestal.

Berglund, B. E. (1979): The Deglaciation of Southern Sweden, 13500-10000. - Boreas, 8: 89-119; Oslo.

Beug, H. J. (1961): Leitfaden der Pollenbestimmung. 63 S., Stuttgart.

Birks, H. J. B. (1973): Post and Present Vegetation of the Isle of Skye. - 415 S., Cambridge.

Bishop, W. W. \& COOPE, G. R. (1977): Stratigraphical and Faunal Evidence for Lateglacial and Early Flandrian Environments in SW Scotland. - in: GRAY, J. M. \& LOWE, J. J. (1977): Studies in the Scottish Lateglacial Environment. - 61-89; Oxford.

Charlesworth, J. K. (1956): The Late-glacial History of the Highlands and Islands of Scotland. - Trans. Royal Soc. Edinburgh, 62: 769-928; Edinburgh.

Clough, C. T. \& Harker, H. (1904): The Geology of West Central Skye with Soay. - Mem. Geol. Surv., London; London. 
Coope, G. R. \& Pennigton,' W. (1977): The Windermere Interstadial of the Late Devension. - Phil. Trans. Royal Soc., 280: 337-339; London.

ERDTMAN, G. (1934): Über die Verwendung von Essigsäureanhydrid bei Pollenuntersuchungen. - Svensk Bot. Tidskr., 28: $354 \mathrm{ff}$; Stockholm.

ERDTMAN, G. (1969): Handbook of Palynology. - 486 S., ; New York.

FAegri, K. \& Iversen, J. (1964): Textbook of Modern Pollen Analysis. - 186 S.; Kopenhagen.

FORBES, J. D. (1845): Notes of the Topography of the Cuchullin Hills in Skye and on Traces of Ancient Glaciers which they present. - Edinburgh New Phil. J., 40: 76-99; Edinburgh.

Gray, J. M. \& Lowe, J. J. (1977 a): The Scottish Lateglacial Environment: A Synthesis. - in: GRAY, J. M. \& LOWE, J. J. (1977): Studies in the Scottish Lateglacial Environment: 163-183; Oxford.

— \& - (1977b): Studies in the Scottish Lateglacial Environment. - $197 \mathrm{~S}$; Oxford.

Gross, G. (1974): Schneegrenzberechnungen an den Gletschern der Silvretta-Gruppe. Systematischer Vergleich der Bestimmungsmethoden. - Geogr. Hausarbeit der Univ. Innsbruck, 126 S.; Innsbruck.

HARKer, A. (1901): Ice-erosion in the Cuillin Hills, Skye. - Trans. Royal Soc. Edinburgh, 40: 221-252; Edinburgh.

HEUBERGER, H. (1966): Gletschergeschichtliche Untersuchungen in den Zentralalpen zwischen Sellrain- und Ötztal. - Wiss. Alpenvereinshefte, 20: 125 S.; Innsbruck.

- (1968): Die Alpengletscher im Spät- und Postglazial. — Eiszeitalter und Gegenwart, 19: 270-75; Hannover.

KAISER, K. (1980): Quartäre Meeresstrände und „Head”Kliffs britischer Küsten; Fortschritte und Probleme der Küstenmorphologie. - Berliner Geogr. Studien, 7: 59-97; Berlin.

KerSCHNER, H. (1976): Das Daun- und das Egesenstadium in ausgewählten Tälern der Zentralalpen von Nordtirol und Graubünden. - Diss. Universität Innsbruck; Innsbruck.

LOWE, J. J. \& Gray, J. M. (1980): The Stratigraphic Subdivision of the Late glacial of NW Europe: A Discussion. — in: LOWE, J. J. et al. (1980): Studies in the Lateglacial of Northwesteurope. - 157-177; Oxford

- \& WAlKeR, J. C. (1980): Problems associated with Radiocarbon Dating the close of the Lateglacial Period in the Rannoch Moor Area. - in: Lowe, J. J. et al. (1980): Studies in the Lateglacial of Northwesteurope. 123-139; Oxford.

- et al. (1980): Studies in the Lateglacial of Northwesteurope. - 205 S.; Oxford.
MacPherson, J. B. (1980): Environmental Change during the Loch Lomond Stadial: Evidence from a Site in the Upper Spey Valley. - in: LOWE, J. J. et al. (1980): Studies in the Lateglacial of Northwesteurope. 89-103; Oxford.

MAISCH, M. (1981): Glazialmorphologische und gletschergeschichtliche Untersuchungen im Gebiet zwischen Landwasser und Albulatal (Kanton Graubünden, Schweiz). - Arbeiten Geogr. Inst. Univ. Zürich; Ser. A., No. 470: 215 S.; Zürich.

MANGERUd, J. (1979): Glacial History of Western Norway, 15000-10000 B. P. - Boreas, 8: 179-187; Oslo.

- (1980): Ice-front Variations of different Parts of the Scandinavian Ice-sheet, $13000-10000$ B.P. - in: LOWE, J. J. et al. (1980): Studies in the Lateglacial of Northwesteurope. - 23-31; Oxford

- et al. (1974): Quarternary Stratigraphy of Norden, a Proposal for Terminology and Classification. - Bores; 8: 109-127; Oslo.

MESSERLI, B. (1967): Die eiszeitliche und die gegenwärtige Vergletscherung im Mittelmeerraum. - Geographica Helvetica, 22: 105-228; Basel.

MÖRNER, N. A. (1969): The Late Quarternary History of the Kattegatt Sea and the Swedish West Coast. Deglaciation, Shorelevel Displacement, Chronology, Isostasy and Eustasy. - Sver. Geol. Unders., C640: $487 \mathrm{ff}$.; Stockholm

PAtzelt, G. (1973): Die neuzeitlichen Gletscherschwankungen in der Venedigergruppe. - Z. Gletscherkde. u. Glazialgeol., 9: 1-2, 5-57; Innsbruck.

- \& Bortenschlager, S. (1973): Die postglazialen Gletscher- und Klimaschwankungen in der VenedigerGruppe. - Z. Geomorph., 16: 28-70; Berlin.

Pennington, W. (1975): A Chronostratigraphic Comparison of Late-Weichselian and Late-Devensian Subdivisions, illustrated by two radiocarbondated Profiles from Western Britain. - Boreas, 4: 157-171; Oslo.

- (1977): Lake Sediments and the Lateglacial Environment in the Northern Scotland. - in: GRAY, J. M. \& LOWE, J. J. (1977): Studies in the Scottish Lateglacial Environment. - 119-143; Oxford.

PrICE, R. J. (1973): Glacial and Fluvioglacial Landforms. - 242 S.; London.

RENNER, F. (1982): Beiträge zur Gletschergeschichte des Gotthardgebietes und dendroklimatologische Analysen an fossilen Hölzern. - Arbeiten Geogr. Inst. Univ. Zürich; Ser. A. No. 475; Zürich.

Richey, J. E. (1961): British Regional Geology: The Tertiary Volcanic District. - 56 S.; Edinburgh.

Sissons, J. B. (1967): The Evolution of Scotland's Scernery. - Edinburgh

- (1974): The Quarternary in Scotland: A Review. Scot. J. Geol., 10: 311-337; Edinburgh. 
Sissons, J. B. (1977): The Loch Lomond Readvance in Southern Skye and some Palaeoclimatic Implications. Scot. J. Geol., 13: 23-36; Edinburgh.

- (1977 a): The Loch Lomond Readvance in the Northern Mainland of Scotland. - in: GraY, J. M. \& Lowe, J. J. (1977): Studies in the Scottish Lateglacial Environment. - 45-60; Oxford.

- (1980): Palaeoclimatic Inferences from Loch Lomond Advance Glaciers. — in: LOWE, J. J. et al. (1980): Studies in the Lateglacial of Northwesteurope. - 31-44; Oxford.

Straka, H. (1975): Pollen- und Sporenkunde. -238 S.; Stuttgart.

VAN HUSEN, D. (1983): Exkursionspunkte zur Route im Gletschergebiet der oberösterreichischen Traun. - Exkursionsführer zur International Union for Quarternary Research, 171-183; München.

VASARI, Y. (1977): Radiocarbon Dating of the Lateglacial and Early Flandrian Vegetational Sucession in the Scottish Highlands and the Isle of Skye. — in: GrAY, J. M. \& LOWE, J. J. (1977): Studies in the Scottish Lateglacial Environment. - 143-162; Oxford.
VASARI, Y. \& VASAR, A. (1968): Late- and Post glacial macrophytic Vegetation in the Lochs of Northern Scotland. - Acta Botanica Fennica, 45: 193-217; Helsinki.

VON HÖFER, H. (1879): Gletscher- und Eiszeitstudien. Sitzber. Ak. Wiss., math.-phys. Kl.; I, 79; Wien.

WALTHER, H. \& LieTH, H. (1960): Klimadiagramm-Weltatlas. $-258 \mathrm{~S}$.; Jena.

WALTHER, M. (1984): Geomorphologische Untersuchungen zum Spätglazial und Frühholozän in den Cuillin Hills (Insel Skye, Schottland). - Diss. Freien Universität Berlin, 192 S.; Berlin.

Wilhelm, F. (1972): Hydrologie, Glaziologie. - 201 S.; Braunschweig.

Manuskript eingegangen am 5. 2. 1986,

Nachträge Dezember 1986. 
